\title{
eJRIEPS
}

Ejournal de la recherche sur l'intervention en éducation physique et sport

$13 \mid 2008$

Varia

\section{Débats d'idées et apprentissage chez les filles en sport collectif}

Zeineb Zerai, Meher Rezig et Makram Zhigbi

\section{(2) OpenEdition}

Journals

Édition électronique

URL : https://journals.openedition.org/ejrieps/6125

DOI : 10.4000/ejrieps. 6125

ISSN : 2105-0821

Éditeur

ELLIADD

Référence électronique

Zeineb Zerai, Meher Rezig et Makram Zhigbi, « Débats d'idées et apprentissage chez les filles en sport collectif », eJRIEPS [En ligne], 13 | 2008, mis en ligne le 01 janvier 2008, consulté le 17 juin 2021. URL : http://journals.openedition.org/ejrieps/6125; DOl : https://doi.org/10.4000/ejrieps.6125

\section{(0) (1)}

La revue eJRIEPS est mise à disposition selon les termes de la Creative Commons Attribution 4.0 International License. 
Débats d'idées et apprentissage chez les filles en sport collectif

Zeineb Zerai, Meher Rezig \& Makram Zhigbi

ISSEP du Kef et Université de Tunis. Tunisie

\section{Résumé}

En sport collectif, la volonté d'associer les élèves à la construction de leurs connaissances et compétences motrices a favorisé l'apparition de temps de "débat d'idées". Ce sont des situations dans lesquelles les élèves s'expriment et débattent à propos du jeu. Cet échange est destiné à faire évoluer ou non le projet d'action de l'équipe en analysant la stratégie prévue et la tactique effectivement appliquée.

Aussi, dans cet article, nous envisageons de revenir sur les concepts utilisés dans le mémoire de Zerai (2006) à propos de l'apprentissage du handball chez des filles Tunisiennes. Le rôle de la cognition dans l'enseignement des sports collectifs suscite des interrogations et fait débat entre les tenants du tout cognitif et les tenants d'une approche plus écologique. Il s'agira de montrer que des données chiffrées relevées à l'occasion d'une rencontre fournissent des indications précieuses concernant les rapports de personne existant dans les équipes. Ainsi on obtient des données fiables qui permettent d'alimenter un débat d'idées. La coopération au sein du groupe permet d'agir sur l'enjeu et la nature de la tâche. Ceci nous permettra de conclure qu'une bonne collaboration entre les joueuses peut avoir des effets positifs sur les apprentissages.

Un des acquis de la recherche en didactique des sports collectifs à l'école est que, si l'apprentissage d'un jeu ne peut se concevoir sans une pratique importante, il nécessite aussi une prise de distance avec l'immédiateté de cette pratique. L'enjeu est de conceptualiser l'action pour construire des invariants opératoires et des règles d'action (Gréhaigne, 1992 ; Vergnaud, Halbwachs \& Rouchier, 1978 ; Wallian \& Gréhaigne, 2004). On n'apprend pas qu'en jouant ou en répétant des gestes techniques ; il est besoin, pour parvenir à des généralisations et à la construction d'un sens, d'analyser après coup ce qui s'est passé. Les travaux actuels sur le " débat d'idées " (Gréhaigne \& Godbout, 1998) en didactique des sports collectifs posent le problème des relations entre apprentissage moteur et verbalisation. Dans cet article, nous envisageons de revenir sur les paradigmes et les concepts utilisés dans le mémoire de Zerai (2006) sur l'apprentissage des filles en handball. Le rôle de la cognition dans l'enseignement des sports collectifs suscite des 
interrogations et fait débat entre les tenants du tout cognitif et les tenants d'une approche plus écologique. Avec une posture épistémologique, caractéristique d'une recherche technologique (Bouthier \& Durey, 1994), il s'agira de montrer que des données chiffrées relevées à l'occasion d'une rencontre fournissent des indications précieuses concernant les rapports de personne existant dans les équipes. Ainsi on obtient des données fiables qui permettent d'alimenter un débat d'idées.

Par rapport à l'utilisation du modèle cognitiviste du Système de Traitement de l'Information (STI) comme modèle objectif, prégnant et exclusif, nous mettrons en avant les modèles alternatifs de l'action située (Suchman, 1987) et de la cognition située et distribuée (Hutchins, 1995). Ils ont été peu exploités encore pour étudier les phénomènes en sport collectif et les prises de décisions du sujet en jeu. De plus, avec la verbalisation, ils nous paraissent constituer le niveau d'explication le plus adéquat pour appréhender la complexité et l'adaptation des décisions prises en jeu.

Après avoir resitué rapidement les pré-supposés et les enjeux de la cognition située et distribuée, nous reviendrons sur les résultats obtenus par Zerai (2006) afin de mieux évaluer les conséquences de ce type d'approche sur le débat d'idées.

\section{La cognition située et distribuée}

L'un des objectifs de ce travail consiste à identifier les relations existant entre les éléments qui interagissent lors des prises de décision dans l'action, en se penchant notamment sur l'organisation fonctionnelle de l'activité du joueur et sa logique propre qui n'est pas toujours rationnelle. (Bouthier, 1993, 2000 ; Mouchet, 2003). Pour mieux comprendre ce qui se joue dans les dialogues des débats d'idées nous allons appuyer sur deux paradigmes complémentaires : l'action située et la cognition distribuée.

\section{1. L'action située}

Suchman (1987) met l'accent sur le caractère profondément social de l'action humaine, celle-ci étant considérée comme un accomplissement pratique, singulier, situé, socialement et culturellement; elle porte l'empreinte du contexte dans lequel elle s'inscrit. «Par action située, je veux simplement dire: prise dans le contexte de circonstances particulières et concrètes. " (Suchman, 1987, p. 28). En ce qui concerne les rapports entre le sujet et l'environnement, l'auteur s'appuie, comme Ochanine (1978), sur l'idée de représentation mentale d'une réalité externe. Ces représentations sont dépendantes de la situation et de la succession des configurations du jeu; une objectivation de l'action n'existe qu'après la situation considérée. Cette dernière est donc prioritaire pour l'analyse de l'action, certes en référence à un contexte relatif à l'état du monde physique, et qui inclut aussi l'histoire sociale dans laquelle s'inscrit le joueur. Néanmoins, une bonne 
solution implique souvent de ne relever que quelques caractéristiques de la configuration, un agencement partiel des éléments qui réunira toutes les relations indispensables et elles seules. Ochanine $(1971 ; 1978)$ appelle cet agencement partiel des éléments « structure opérative ", et " image opérative " sa représentation chez le sujet. C'est une image " économique », réduite aux éléments indispensables qui a comme principales propriétés l'adaptation à l'opposition, le laconisme, la plasticité et un caractère intentionnel... Cette image, reflet fonctionnel finalisé, est constituée par et pour l'activité du joueur.

En ce qui concerne les rapports entre cognition et action, l'activité cognitive est donc mobilisée dans et par cette action ; elle a une spécificité liée au contexte et doit donc être étudiée en situation (Suchman, 1987). Cette primauté de l'interprétation en situation, n'exclut pas les connaissances préalables mais c'est la situation qui organise prioritairement l'action, car elle contient les connaissances premières nécessaires à la continuité ou discontinuité du jeu (Mouchet, 2005).

Dans ce cadre conceptuel, nous considérons que les décisions résultent rarement de l'application de planifications préalables, mais sont construites en jeu, en fonction des interactions locales entre le contexte et les actions des joueurs. Si nous mettons ainsi en valeur le caractère situé, singulier, circonstancié des décisions, il convient de ne pas évacuer aussi leur caractère situé dans un contexte social et culturel plus général.

\section{2. La cognition distribuée}

La cognition distribuée (Hutchins, 1995) constitue un cadre complémentaire dans l'appréhension des activités socialement organisées. En rapport avec les données présentées dans le paragraphe précédent, cette conception réhabilite le rôle du fonctionnement social, et de la culture, dans l'étude de la cognition humaine. Dans un débat d'idées, l'analyse de la lecture du jeu ne s'opère pas isolément du contexte social qui la voit se déployer et les savoirs émergent dans une situation d'interaction sociale dans laquelle les échanges avec des pairs prennent tout leur poids (Vygotsky, 2000). Rey (2003) nuance un peu ce point de vue en nous prévenant qu'intervenir sur une équipe ou un groupe suppose parfois de se débarrasser des cadres conceptuels classiques et traditionnels qui magnifient l'idée qu'une équipe qui réussit se fonde sur l'abnégation, la solidarité, la cohésion, le projet commun. II ne faut pas pour autant nier l'importance des impacts de ces phénomènes sur les performances de l'équipe.

Les travaux menés dans des classes considérées comme des «systèmes cognitifs globaux » (Hutchins, 1995) où la cognition est distribuée entre les acteurs et les objets mettent ainsi en évidence l'émergence de modes d'organisation typiques dans les interactions avec des élèves et/ou des joueurs, ayant des fonctions et des rôles différents. La cognition relève bien d'une « intelligence distribuée » (Hutchins, 1995) : les moyens de 
conception et d'exécution de l'action ont la particularité d'une part de se présenter en partie au moment de l'action, d'autre part de se répartir « dans la tête » des acteurs lors du « débat d'idées ». Aussi bien au niveau individuel que collectif, des contraintes fortes s'appliquent aux processus cognitifs : rien ne peut-être fait par des individus en dehors de ce qu'ils savent faire seuls ou en interaction avec les autres ; rien ne peut être anticipé en dehors de ce qu'ils savent prédire seuls ou en interaction avec d'autres. Dans le débat d'idées, une information touche certains joueurs puis son contenu est traité par le réseau social de l'équipe, produisant d'autres informations par des interactions en chaîne. Ce processus de cognition sociale peut alors donner lieu ou pas à une transformation de la façon de jouer de l'équipe.

\section{L'équipe, le débat et le réseau de compétences}

L'équipe est un espace social qui mobilise des affects, des représentations et qui génère des expressions verbales et non-verbales. Cet ensemble fait émerger une trame de relations d'où surgissent des affinités, des tensions, des images de soi et du groupe. Les dimensions comparatives introduites par les confrontations compétitives sont des moments de comparaison sociale, de jugement, d'évaluation, de différenciation et de valorisation sociale. Surtout, c'est un temps fort de remise en cause de l'identité et de l'estime de soi. Un autre élément est que cette complexité ne conduit pas les joueurs d'une équipe à définir tous, de la même manière, leur appartenance à l'équipe. C'est ce que nous apprend la psychologie sociale des groupes. Chaque membre définit toujours sa manière d'appartenir au groupe.

Dans un groupe / une équipe, il existe des individus :

- qui fonctionnent ensemble, sur la base d'une activité de coopération ;

- qui sont engagés dans une production commune (projets, buts, objectifs) ;

- qui sont conscients de partager une appartenance commune (groupe organisé statutaire).

Redéfinissant ainsi les motivations et les projets du joueur, il semble que le professeur expérimenté a compris que ce qui compte, ce n'est pas de réitérer constamment l'importance de la motivation pour réussir. Ce qui devient important, c'est aussi la motivation d'un joueur plus "situé", c'est l'attention qu'il faut porter aux relations sociales qui sont à l'origine de la motivation et des projets de l'élève.

\section{1. Le débat d'idées}

Dans un débat d'idées, la prise conscience d'une situation en groupe devrait permettre de donner aux faits observés une interprétation cohérente, d'une part et anticiper de futurs états de ces éléments, d'autre part. Les termes d'image mentale, de représentation, ou de 
schémas, renvoient tous à des productions cérébrales modélisant le monde qui entoure le joueur, partie prenante d'une équipe.

Selon Casetti, Lumbelli \& Wolf (1981), le débat est une discussion organisée, une confrontation d'opinions à propos d'un objet particulier mais qui se déroule dans un cadre préfixé. Ainsi, sont en partie prédéterminés la longueur du débat, la durée, le nombre des participants et l'objet de l'échange. De plus un débat comporte généralement un « modérateur » chargé de veiller à son bon déroulement. Le débat tient donc à la fois de la discussion par son caractère descriptif et argumentatif et de l'interview par son caractère médiatique. Le débat fonctionnerait comme une sorte de modèle de la conversation, de miroir idéalisé, démontrant ou s'efforçant de démontrer l'efficacité d'un échange discipliné et que d'une bonne application des règles conversationnelles peut "jaillir la lumière". Dans ce cadre, le débat d'idées constitue une pièce centrale d'une conception constructiviste de l'apprentissage des sports collectifs. II consiste après une séquence jouée et après le retour d'information chiffrée en une discussion destinée à faire évoluer ou non le projet d'action de l'équipe en revenant sur la stratégie prévue et en analysant la tactique appliquée. Des éléments concernant l'utilisation de l'espace de jeu peuvent également fournir des indications précieuses.

\section{2. Le réseau de compétences}

Dans sa classification des activités sportives, Jeu (1977) range les sports collectifs dans la catégorie « compétition » qu'il caractérise ainsi « de l'égalité des chances au départ à l'inégalité du résultat ». Cette définition reste très générale, mais elle met en évidence de façon très explicite les préalables à l'affrontement et la finalité du jeu. Plus précisément, c'est un groupe de joueurs qui affronte un autre groupe de joueurs en se disputant ou en s'échangeant le plus souvent une balle. Ce qui se transforme entre le début et la fin d'une phase de jeu, est le rapport des forces entre les deux équipes afin de marquer dans la cible de l'adversaire tout en protégeant la sienne. Les positions successives et les mouvements respectifs des joueurs et du ballon sont pris en compte pour caractériser ce rapport d'opposition. L'analyse d'une action en sport collectif nécessite par conséquent le repérage et l'analyse des configurations momentanées, initiales et finales, ainsi que des transformations successives afin de pouvoir examiner l'évolution du rapport de forces, les compétences motrices utilisées et les choix tactiques ainsi que stratégiques individuels et collectifs. Ces configurations momentanées correspondent aux rapports d'opposition évolutifs qu'expriment, en particulier, les rapports de vitesse observés entre les joueurs. Dans ce cadre, en fonction d'un rapport de forces, s'instaure, dans chaque équipe, une distribution des tâches ou une répartition tacite des rôles et des fonctions, voire des conflits, que nous appelons « réseau de compétences » (Gréhaigne, Godbout \& Bouthier, 
1999). Celui-ci est constitué par des conduites et des comportements qui peuvent être identifiés chez les élèves, à l'intérieur d'une équipe, en fonction d'un rapport de forces. L'ensemble de ces conduites varie suivant les joueurs, les moments, les facteurs extérieurs et les sports collectifs. La fonction que le joueur se donne ou qui lui est attribuée dans le groupe constitue bien souvent une caractéristique de sa position dans la dynamique de l'équipe (cf. Gréhaigne et al.,1999). Pour évoluer, cette répartition des tâches demande du temps, l'établissement d'un bilan pour chaque joueur en vue d'optimiser les apports de tous au collectif et tout en faisant que la collectivité permettent à tous de s'améliorer

Pour obtenir des données sur ce qui se passe effectivement en jeu et dans le réseau de compétences, on peut analyser les différentes séquences de jeu (Dugrand, 1985 ; Gréhaigne, 2003 ; Gréhaigne, Caty \& Wallian, 2004 ; Gréhaigne, Godbout, \& Bouthier, 1997). Celles-ci peuvent être définies comme les "échanges de balles" entre les joueurs depuis l'entrée en possession du ballon jusqu'à sa perte (but ou balle à l'adversaire) par l'équipe. Par exemple dans une équipe de quatre joueurs, on relève tous les échanges de balles à l'intérieur de chaque équipe : en un mot, on enregistre tous les événements du jeu. On peut repérer précisément le joueur qui fait les engagements et les remises en touche, celui qui perd des balles ou procure des balles à l'équipe en regagnant la possession du ballon, enfin ceux qui tire ou non au but. De plus, le volume de jeu exprime la capacité du joueur ou d'une équipe à jouer pleinement un match dans le sens quantitatif du terme. II rend compte de sa présence et de son activité dans l'affrontement. II se traduit tout simplement par le nombre de balles jouées (BJ) dans un rapport de forces précis.

\section{Expérimentation à I'ISSEP du Kef}

Dans cette expérimentation qui doit être considérée comme une première approche, nous avons choisi de nous familiariser avec les problèmes de recherche en utilisant un groupe restreint et en nous penchant d'abord sur des aspects qualitatifs et quantitatifs.

\section{1. Méthodologie}

La méthodologie employée a consisté à observer une classe d'étudiantes de première année de l'institut supérieur du sport et d'éducation physique du Kef. Dans cette classe, nous avons constitué deux groupes : dix filles pour constituer les deux équipes qui vont jouer ; dix filles en vue des tâches d'observation pour lesquelles elles ont été entraînées pendant deux semaines qui ont précédé le début du cycle de travail. Les fiches d'observation ont été construites à partir de la fiche individuelle d'évaluation proposée par Gréhaigne, Billard \& Laroche (1999). Après le premier test, une vérification de l'exactitude des relevés a été effectuée entre les données obtenues en direct et les données relevées 
en différé à partir de la bande vidéo. Il n'y avait quasi pas d'erreur le jeu étant relativement lent et facile à observer. La fidélité inter observateur peut être estimée comme dans d'autres publication à .90 (cf. Gréhaigne et al.,1997).

Celles qui ont joué ont suivi un cycle de travail de dix séances de 45 minutes. Chaque séance était composée de différentes situations sous forme de jeux au cours desquels les filles jouaient au hand-ball en 5 contre 5 avec deux gardiennes de but. La durée totale du jeu dans les trois tests était de 30 min pour permettre un investissement total des jeunes filles. Le groupe A (contrôle) était donc constitué de cinq joueuses et de cinq observatrices. Les filles ont été confrontées à la modalité " entraînement + consignes ». Les consignes, sous forme de règles des actions efficaces, ont été données au début et cours du jeu. II n'y a pas eu de dialogue entre les joueuses et les observatrices, celles-ci relevant uniquement les données chiffrées pour la recherche. Le groupe B (expérimental) était aussi constitué de cinq joueuses et de cinq observatrices et celles-ci ont été confrontées à la modalité « entraînement + consignes + débat d'idées ». Le débat d'idées a eu lieu entre chaque situation de jeu.

Les séances $n^{\circ} 1, n^{\circ} 5$ et $n^{\circ} 10$ ont été filmées et les dialogues enregistrés à l'aide d'un caméscope.

3. 2. Les données chiffrées

Les données chiffrées figurant dans les tableaux I, II et III ont été obtenues à partir de trois rencontres opposant une équipe $A$ et une équipe $B$ au début du cycle de travail au milieu et à la fin après dix leçons.

Tableau I. Première rencontre BJ total $=314$

\begin{tabular}{|l|l|l|l|l|l|l|l|l|l|l|}
\hline & \multicolumn{3}{|l}{ Équipe A } & \multicolumn{1}{l|}{ Équipe B } \\
\cline { 2 - 11 } & Ja1 & Ja2 & Ja3 & Ja4 & Total & Jb1 & Jb2 & Jb3 & Jb4 & Total \\
\hline $\begin{array}{l}\text { Balles } \\
\text { jouées }\end{array}$ & 40 & 31 & 43 & 70 & 174 & 49 & 53 & 47 & 70 & 240 \\
\hline $\begin{array}{l}\text { Balles } \\
\text { conquises }\end{array}$ & 15 & 10 & 17 & 19 & 61 & 12 & 9 & 8 & 13 & 42 \\
\hline $\begin{array}{l}\text { Balles } \\
\text { perdues }\end{array}$ & 11 & 10 & 13 & 8 & 42 & 19 & 17 & 11 & 14 & 61 \\
\hline Tirs/Buts & 2 & 2 & 4 & 7 & $15 / 4$ & 8 & 7 & 8 & 11 & $34 / 5$ \\
\hline
\end{tabular}


Tableau II. Deuxième rencontre BJ total $=495$

\begin{tabular}{|l|l|l|l|l|l|l|l|l|l|l|}
\hline & \multicolumn{3}{|l}{ Équipe A } & \multicolumn{10}{l|}{ Équipe B } \\
\cline { 2 - 11 } & Ja1 & Ja2 & Ja3 & Ja4 & Total & Jb1 & Jb2 & Jb3 & Jb4 & Total \\
\hline $\begin{array}{l}\text { Balles } \\
\text { jouées }\end{array}$ & 37 & 30 & 39 & 55 & 161 & 89 & 76 & 79 & 90 & 334 \\
\hline $\begin{array}{l}\text { Balles } \\
\text { conquises }\end{array}$ & 12 & 8 & 10 & 17 & 46 & 15 & 15 & 17 & 20 & 67 \\
\hline $\begin{array}{l}\text { Balles } \\
\text { perdues }\end{array}$ & 20 & 13 & 15 & 19 & 67 & 14 & 13 & 7 & 12 & 46 \\
\hline Tirs/Buts & 1 & 2 & 4 & 6 & $13 / 7$ & 3 & 8 & 8 & 9 & $28 / 11$ \\
\hline
\end{tabular}

Tableau III. Troisième rencontre BJ total $=549$

\begin{tabular}{|l|l|l|l|l|l|l|l|l|l|l|}
\hline & \multicolumn{3}{l|}{ Équipe A } & \multicolumn{3}{l|}{ Équipe B } \\
\cline { 2 - 11 } & Ja1 & Ja2 & Ja3 & Ja4 & Total & Jb1 & Jb2 & Jb3 & Jb4 & Total \\
\hline $\begin{array}{l}\text { Balles } \\
\text { jouées }\end{array}$ & 35 & 28 & 37 & 50 & 150 & 100 & 99 & 90 & 110 & 399 \\
\hline $\begin{array}{l}\text { Balles } \\
\text { conquises }\end{array}$ & 2 & 5 & 7 & 9 & 23 & 19 & 17 & 20 & 25 & 81 \\
\hline $\begin{array}{l}\text { Balles } \\
\text { perdues }\end{array}$ & 25 & 16 & 18 & 22 & 81 & 7 & 6 & 5 & 5 & 23 \\
\hline Tirs/Buts & 0 & 1 & 4 & 4 & $9 / 5$ & 1 & 6 & 7 & 8 & $22 / 16$ \\
\hline
\end{tabular}

Ces différents nombres nous informent sur l'évolution de la circulation de balle entre les partenaires ainsi que le nombre d'échanges de balle réussis: plus le nombre de balles jouées est important plus le jeu est de qualité dans une unité de temps donné. Concernant notre étude, on remarque une amélioration au niveau de la conservation de la balle pour le groupe B. Cette amélioration est croissante durant tout le cycle d'apprentissage. Après cinq séances d'apprentissage, le nombre de balles jouées est passé de 240 durant le premier match à 334 pour le deuxième pour atteindre en fin 399 au cours du troisième match. Le nombre de balles jouées diminuent pour l'équipe $A$

Lors de la première rencontre, la conservation de la balle a été très difficile dans le demiterrain offensif car, dans cet espace, le rapport de forces est souvent en faveur des défenseurs. Après cinq séances d'entraînement, avec un débat d'idées qui accompagne chaque situation, le nombre d'échange de balles jouées augmente progressivement. 
À la dixième séance, le nombre d'échanges de balles devient plus important grâce à une amélioration du niveau des solutions proposées suite à une prise de décision collective planifiée et argumentée et à un débat d'idées qui produit des réponses mieux appliquées.

3. 3. Réseau de compétences et données chiffrées.

Nous allons illustrer comment on peut obtenir des informations sur le fonctionnement du réseau de compétence à l'aide de données chiffrées.

3. 3. 1. Les balles jouées dans l'équipe $B$

La figure 1 présente la distribution des balles jouées dans l'équipe $B$ lors de la première et de la dixième rencontre. On constate tout d'abord une augmentation du nombre de balles jouées ce qui traduit une amélioration du niveau de jeu (le ballon est plus longtemps en jeu). Par contre la distribution des balles entre les joueuses est restée relativement constante. Jb4 semble mener le jeu.

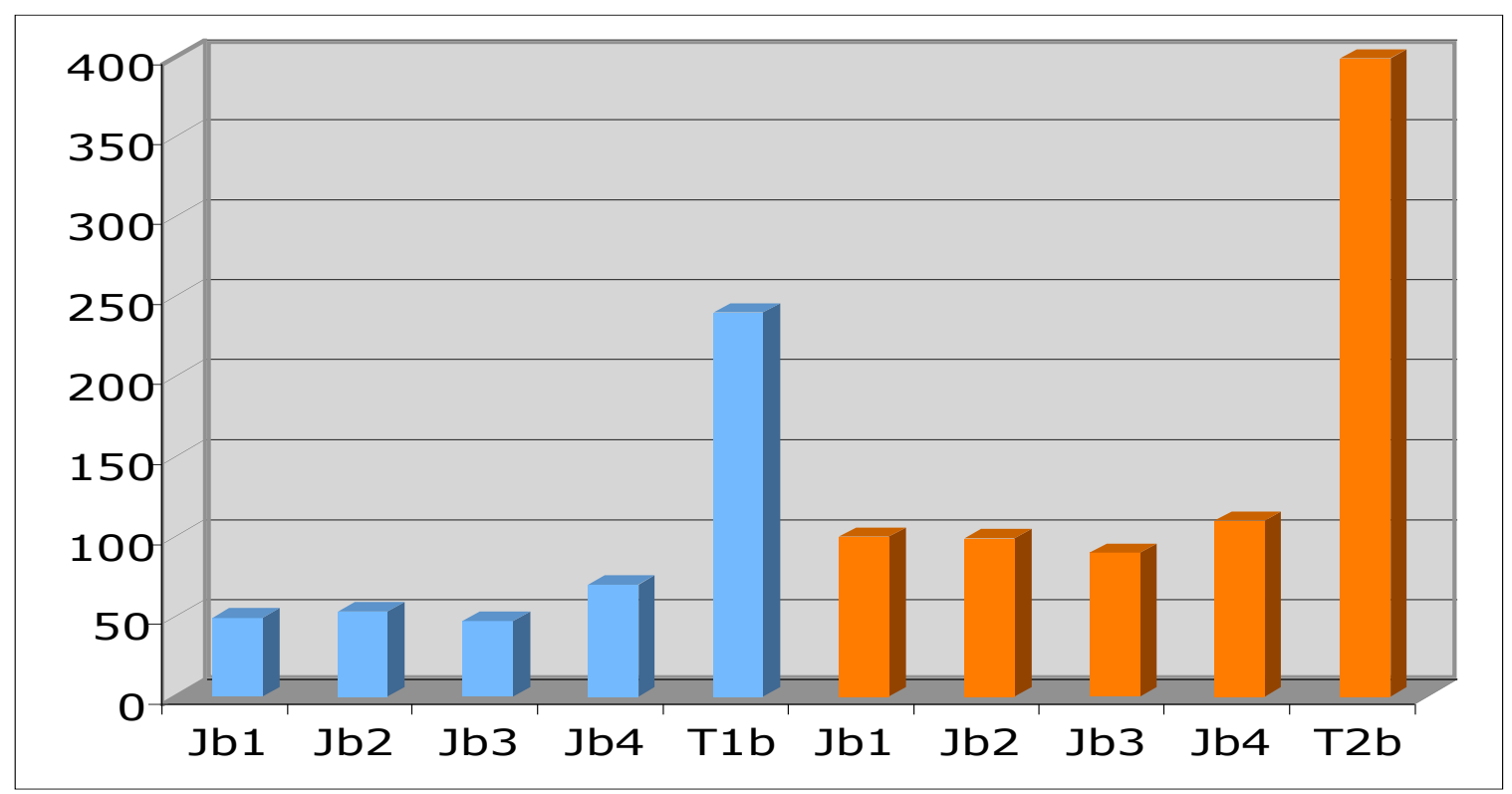

Figure 1. Répartition des balles jouées dans l'équipe $B$ lors de la première et dernière rencontre.

\section{3. 2. Les balles jouées dans l'équipe $A$}

Pour l'équipe A, la figure 2 illustre la répartition des balles jouées lors de la première et de la dernière rencontre. On constate, ici, tout d'abord une diminution du nombre de balles jouées ce qui souligne une domination importante de l'équipe B lors de leur affrontement. La distribution des balles entre les joueuses reste relativement constante. Ja 4 semble mener le jeu. 


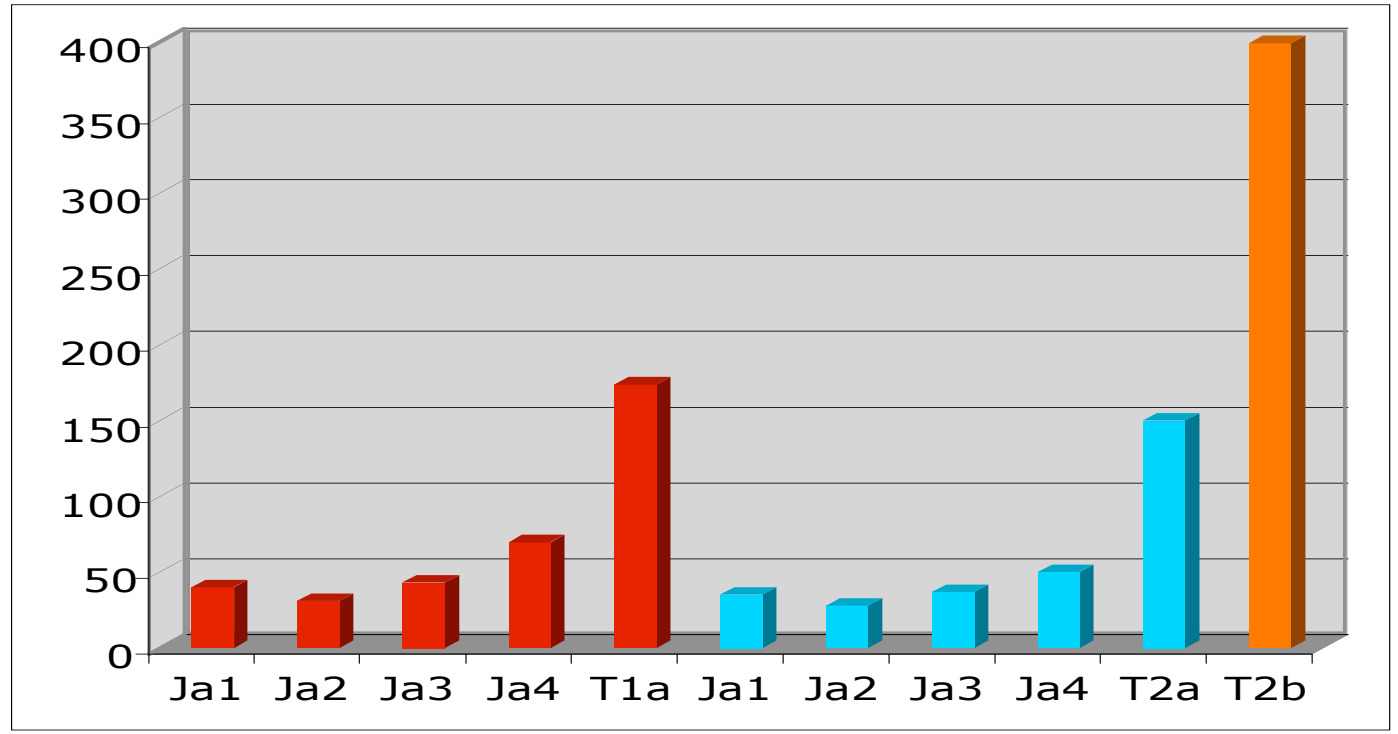

Figure 2. Balles jouées dans l'équipe A lors de la première et dernière rencontre. Une comparaison est possible avec les balles jouées de l'équipe B lors du dernier match.

3. 3. 3. Balles conquises

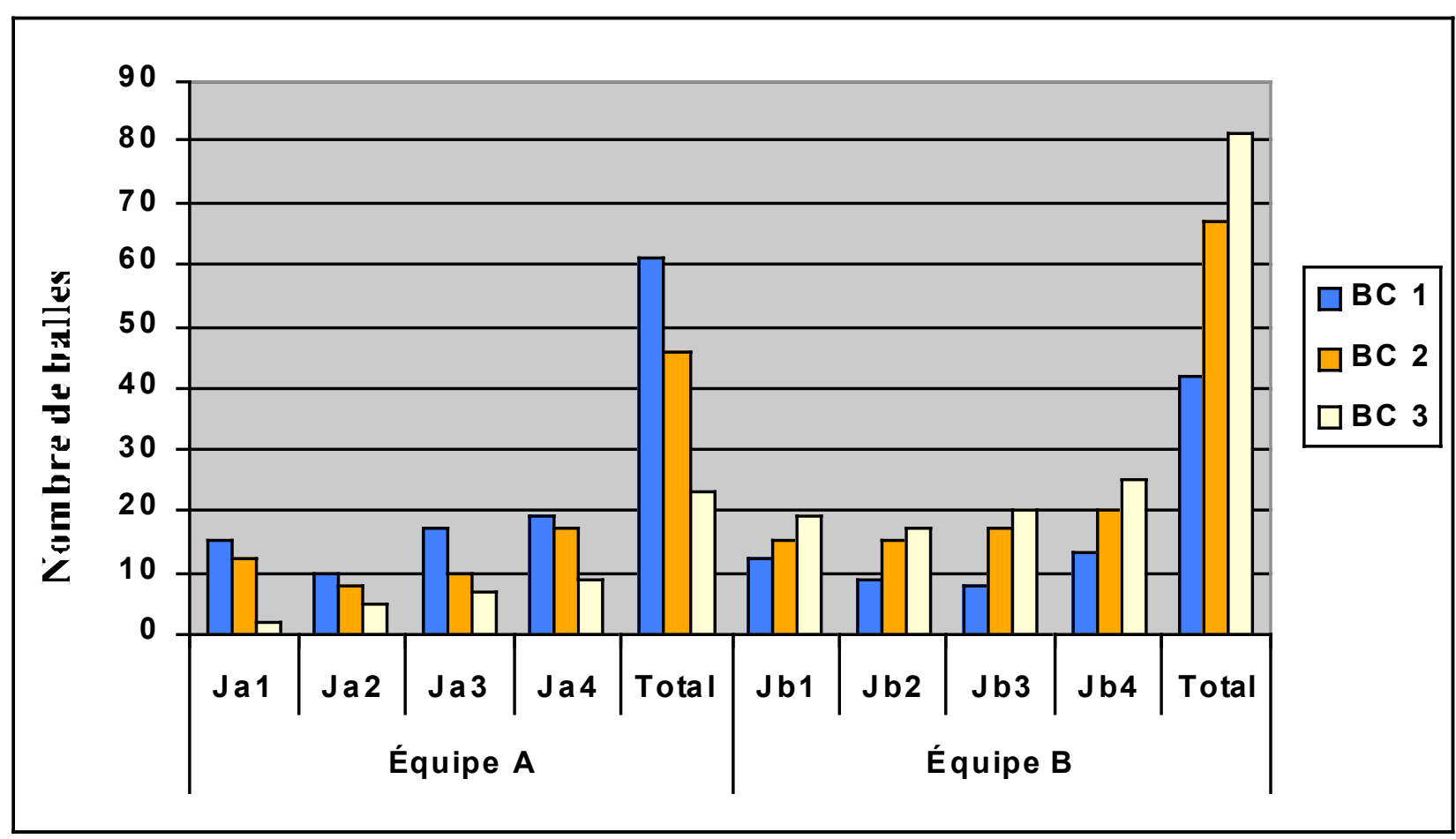

Figure 3. Les balles conquises lors des trois rencontres.

Les balles conquises $(\mathrm{BC})$ constituent, d'abord, le témoignage pour un joueur donné de ses compétences et de son engagement au niveau défensif en comptabilisant la quantité de balles gagnées sur l'adversaire. Le joueur entre en possession de la balle soit grâce à un duel, soit par une interception ou une violation du règlement. Notons que si le besoin 
s'en fait sentir, on peut créer dans les balles conquises, une catégorie remise en jeu, dont on ne tient compte que pour certains joueurs afin d'éviter des biais dans le relevé des balles conquises avec ceux qui font systématiquement les touches et les coup-francs.

La figure 3 illustre la répartition des balles conquises durant les trois rencontres pour les deux groupes. Pour le groupe B le nombre des balles conquises passe de 42 à 67 pour atteindre 81 lors de la dernière rencontre. Cette croissance souligne la progression du niveau d'organisation et de la lecture de jeu (efficacité de la décision prise de point de vue planification mise en place...) ; ainsi que sur la pression exercée par le groupe B sur le groupe $A$; la répartition des tâches au sein du groupe même reste relativement constante et c'est la joueuse $\mathrm{Jb} 4$ qui mène toujours le jeu et qui récupère le plus de ballons. L'interception de la balle constitue un moment clé dans la situation à double effet (Deleplace, 1979). A l'instant précis où la balle change de camp, les rôles se renversent, les filles en défense deviennent des attaquantes et vice versa, alors un nouveau jeu s'établit. Le choix de récupérer la balle dans son demi terrain offensif devient alors un élément tactique important, le chemin pour aller au but étant plus court.

Avec la répartition des balles perdues et des balles conquises par demi terrain (Zerai \& Gréhaigne, 2007) nous avons peut être une explication au fait que l'équipe $A$ a perdu beaucoup de ballons dans son demi terrain défensif permettant ainsi à l'équipe $B$ de récupérer la balle dans des conditions favorables et de marquer

3. 3. 4. Capacités offensives: nombre de tirs, nombre de buts dans l'équipe $B$

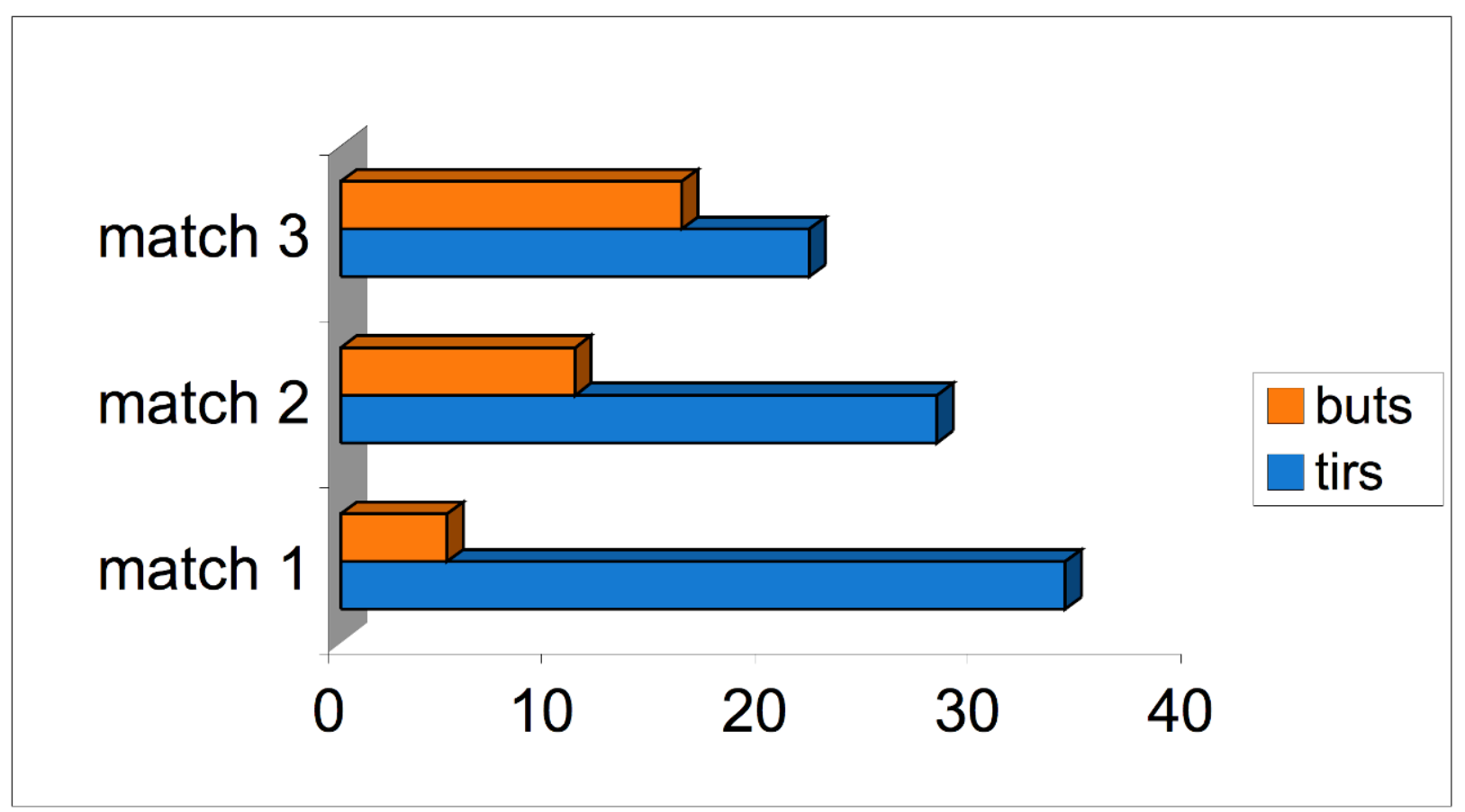

Figure 4. Capacité offensive : nombre de tirs et buts pour l'équipe B. 
Durant les trois compétitions, le nombre de tirs évolue comme suit : 34,28 et puis 22 alors que le nombre de buts augmente : 5, 11 et 16 . Si on analyse le nombre de tirs et le nombre de buts, on constate qu'ils fonctionnement en sens inverse ; en d'autre terme, le nombre de tirs décroît alors que le nombre de buts augmente soulignant les progrès dans la phase de réalisation.

\section{4. Commentaires}

Ces résultats soulignent bien que la notion de rôle (conduite qui exprime ce que le joueur pense avoir à faire en rapport avec la façon dont il vit le rapport de forces, le réseau de compétences dans l'équipe avec la façon dont il gère ses ressources dans ce système de contraintes) est essentielle à l'analyse d'une rencontre. A l'interface de la logique du sujet, de la logique du groupe et de la logique interne du sport considéré, la place du joueur dans ce réseau de compétences constitue, souvent, un révélateur fiable des rapports réciproques entre ce joueur et l'équipe. Pour évoluer, cette répartition des tâches demande du temps, l'établissement d'un bilan pour chaque joueur en vue d'optimiser les apports de tous au collectif et tout en faisant que la collectivité permettent à tous de s'améliorer. Ce postulat renvoie aussi bien à mieux utiliser les points forts d'une équipe, qu'à revoir quelques "manques criants". En un mot, c'est fondamentalement s'appuyer sur ce que les gens savent faire pour amorcer, développer et stabiliser les apprentissages de tous et de chacun.

Ce travail montre bien l'importance d'une investigation contextuelle et sociale afin d'approfondir la compréhension du fonctionnement des équipes de jeu sportif collectif, véritables groupes sociaux qui s'affrontent de façon rituelle. En effet, souvent la dimension émotionnelle des jeux collectifs produit chez les filles en déficit de jeu et d'apprentissage une « survalorisation » de l'équipe et de la dimension collective dans les débats d'idées. Les joueuses ont l'air de penser que le collectif va les sauver!

\section{Discussion}

Dans notre expérimentation, l'analyse des comportements des joueuses n'ayant pas eu de débat d'idées (groupe A) montre une progression au niveau technique, une application directe des consignes sans même les interpréter ou les adapter aux caractéristiques du jeu. Les décisions prises en jeu ne semblent ni planifiées ni guidées par un projet. Les élèves ont des difficultés à se passer la balle et même à la récupérer: la plupart du temps, les filles n'arrivent pas à atteindre la zone de 9 mètres, la balle étant interceptée dans l'entre jeu. En conséquence, la conservation de la balle est brève suite à des fautes de règlement (marché, passage en force). 
Dans l'autre groupe, l'observation des résultats réalisés par les jeunes filles avant le débat d'idées est de même nature que la description ci-dessus. Après un débat d'idées, on remarque un changement au niveau de l'organisation du jeu à savoir une montée de balles rapide et organisée. Les passes sont précises. Selon le déroulement du jeu, les joueuses se trouvent en appui ou en soutien du porteur de balles. Souvent, elles créent l'incertitude chez l'adversaire par un renversement brusque du jeu en envoyant l'adversaire d'un côté alors que la balle est envoyée de l'autre côté. En défense, un retour rapide en zone après une perte de balle représente aussi un nouvel apprentissage. Les remises en jeu sont rapides, les tirs sont mieux ciblés.

L'interaction verbale qui a eu lieu à propos des situations d'apprentissage met à jour pour les apprenants l'existence d'autres points de vue qui peuvent être contradictoires avec leurs propres idées. Pourtant, les réponses proposées peuvent être plus efficaces, enrichissant ainsi le répertoire des réponses disponibles. Le débat d'idées facilite pour les filles l'acceptation de l'avis de l'autre. En effet, le débat, l'analyse, le fait de défendre sa réponse par rapport au problème posé par le jeu aboutissent à une réflexion collective. Ce type de travail est basé sur une co-construction des connaissances à partir d'actions partagées. Dans ces échanges, des désaccords apparaissent. Pour les dépasser les jeunes filles doivent établir des liaisons logiques entre les différentes propositions, coordonner leurs idées pour enfin construire un nouveau plan d'action collectif. Après, avec le retour au jeu, on constate souvent un progrès qui se traduit par une diminution des fautes commises, par moins de pertes de balles et par plus de buts marqués. Ces premiers résultats semblent prouver l'utilité d'interactions verbales lors des apprentissages. Leurs effets sur la construction des connaissances et des compétences à propos du jeu sont également à noter.

La différence au niveau des apprentissages entre les deux groupes met en lumière l'efficacité de cette approche pédagogique dans la construction des connaissances. En effet, on n'applique plus des recettes, des solutions à reproduire en face d'un problème. Une analyse du contexte, des interactions avec autrui, des confrontations d'idées mobilisent les activités cognitives des apprenants. La méthodologie de débat d'idées constitue l'occasion de mettre en place, à partir d'un problème posé par le jeu, une réflexion collective qui amène, souvent, une amélioration des connaissances mais également des compétences motrices.

\section{Conclusion}

Par une meilleure conscience de ce que l'on a à faire, les interactions verbales entraînent une réduction de l'incertitude vis-à-vis des partenaires. La prise en compte de l'adversaire, 
un plan de jeu bien établi avec ses partenaires et une juste évaluation de ses propres capacités favorisent une amélioration du niveau tactique. Les joueuses ayant bénéficié d'interactions verbales obtiennent de meilleurs résultats que ceux de l'autre groupe n'ayant pas inter-agi verbalement. II se confirme que pour progresser, les filles font appel à des connaissances antérieures pour argumenter dans le débat. Ensuite la coopération au sein du groupe permet d'agir sur l'enjeu et la nature de la tâche. Ceci nous permet de conclure que l'apprentissage coopératif peut avoir des effets positifs sur l'estime de soi et les relations d'équipe.

La verbalisation facilite bien l'apprentissage et le rend plus efficace. Souvent, l'interaction au sein du groupe repose sur un conflit. Ce conflit est une source de progrès car il permet aux filles qui se trompent de prendre conscience de leurs erreurs et de l'existence de solutions alternatives. La fonction perturbatrice du partenaire peut se manifester dans le désaccord et l'argumentation mais également en ouvrant le champ des possibles. En effet il n'existe pas une solution unique mais des solutions pertinentes en fonction du déroulement de la situation.

Nous aurons sans doute l'occasion d'y revenir, dans une prochaine expérimentation.

\section{Bibliographie}

Bouthier, D. (1993). L'approche technologique en S.T.A.P.S. : représentations et actions en didactique des APS. Diplôme d'habilitation à diriger des recherches. Orsay : Université Paris-Sud.

Bouthier, D. (2000). La coordination des décisions individuelles; contribution de l'intelligence tactique. Colloque Préparation Olympique L'évolution de la pensée tactique, Noisy le Grand, 14 et 15 novembre 2000.

Bouthier, D., \& Durey, A. (1994) Technologie des activités physiques et sportives. Impulsions 1, 117-126.

Casetti, F., Lumbelli, L, \& Wolf, M. (1981). «Indagine su alcune regole di genere televiso », Ricerche sulla communicazione. Quaderni semestrali dell'Istituto A. Gemeli Franco Angeli ${ }^{\circ} 3$, Milan : Ikon, 11-122.

Deleplace, R. (1979). Rugby de mouvement, rugby total. Paris : Revue EPS.

Dugrand, M. (1985). Approches théorique, expérimentale et clinique de l'enseignement du football. L'exemple au Sénégal. Thèse de troisième cycle, Université de Caen.

Gréhaigne, J. F. (1992). L'organisation du jeu en football. Paris : ACTIO. 
Gréhaigne, J.F.(2003). Vers une didactique constructiviste en sport collectif. In C. Amade-

Escot, (Ed.), Didactique de l'éducation physique : États des recherches (pp. 79102). Paris : Revue EPS.

Gréhaigne, J.F., \& Godbout, P. (1998). Observation, critical thinking and transformation :

Three key elements for a constructivist perspective of the learning process in team

sports. In R. Feingold, R. Rees, G. Barrette, L. Fiorentino, S. Virgilio, \& E.

Kowalski (Eds.), Education for life (pp. 109-118). New York : Adelphi University.

Gréhaigne, J.F., Caty, D., \& Wallian, N. (2004). Évaluation authentique et rapport de forces en sport collectif. Mesures et Evaluation en Education, 27 (3), 25-39.

Gréhaigne, J.-F., Godbout, P., \& Bouthier, D. (1997). Performance assessment in team sport. Journal of Teaching in Physical Education, 16, 500-516.

Gréhaigne, J.F., Godbout, P., \& Bouthier, D. (1999). The foundations of tactics and strategy in team sports. Journal of Teaching in Physical Education, 18, 159-174.

Hutchins, E. (1995). Cognition in the wild. Cambridge : M.I.T. Press.

Jeu, B. (1977). Le sport, l'émotion, l'espace. Paris : Vigot.

Mouchet, A. (2003). Caractérisation de la subjectivité dans les décisions tactiques des joueurs d'Elite 1 en rugby, Thèse pour le Doctorat de l'Université Bordeaux 2, non publiée.

Mouchet, A. (2005). Modélisation de la complexité des décisions tactiques en rugby. eJRIEPS, 7, 03-15.

Ochanine, D. (1971). L'image opérative effectrice. Questions de Psychologie, 3.

Ochanine, D. (1978). Le rôle des images opératives dans la régulation des activités de travail. Psychologie et Education, 2, 63-72.

Rey, J.P. (2003). L'expertise dans la gestion de 1'équipe de jeu sportif collectif : quel regard de la psychologie sociale ? Les cahiers de I'INSEP, 35, 30-33.

Suchman, L. (1987). Plans and situated actions: The problem of communication. Cambridge : Cambridge University Press.

Vergnaud, G , Halbwachs, F., \& Rouchier, A. (1978). Structure de la matière enseignée, histoire des sciences et développement conceptuel chez l'enfant. Revue Française de Pédagogie, 45, 7-18.

Vygotski, L. (2000). Pensée et langage. Paris: La Dispute.

Wallian, N., \& Gréhaigne, J.F. (2004). Vers une approche sémioconstructiviste des apprentissages moteurs. In G. Carlier (Ed.), Si l'on parlait du plaisir d'enseigner l'éducation physique (pp. 167-179). Montpellier : AFRAPS 
Zerai, Z. (2006). Comment les filles apprennent-elles en Hand Ball ? Apport de la verbalisation. Mastère en didactique des Activités Physiques et Sportives. Tunis, ISEFC.

Zerai, Z., \& Gréhaigne, J.F. (2007). Quelques aspects de la cognition située et distribuée dans l'apprentissage des sports collectifs. Présentation orale au Congrès CEM 07 : Cognition, Emotion \& Motivation, 26, 27 et 28 octobre 2007. Hammamet, Tunisie. 